\title{
Morbimortalidad cardiopulmonar en pacientes con lupus eritematoso sistémico
}

\author{
Alicia Aquino Valdovinos ${ }^{\mathrm{I}}$, María Isabel Acosta Colmán ${ }^{\mathrm{I}}$, Osmar Antonio Centurión ${ }^{\mathrm{II}}$, Gabriela Ávila \\ Pedretti ${ }^{\mathrm{I}}$, Jhonatan Losanto ${ }^{\mathrm{I}}$, Margarita Duarte ${ }^{\mathrm{I}}$ \\ I. Departamento de Reumatología. Hospital de Clínicas. Universidad Nacional de Asunción \\ II. Primera Cátedra de Clínica Médica. Hospital de Clínicas. Universidad Nacional de Asunción
}

Cómo referenciar este artículo/ How to reference this article:
Aquino A, Acosta MI, Centurión OA, Avila G, Losantos J, Duarte M. Morbimortalidad cardiopulmonar en pacientes con lupus eritematoso sistémico. Mem. Inst. Investig. Cienc. Salud. $2015 ; 13(2): 127-38$

\section{R E S U M E N}

Los pacientes con diagnóstico de lupus eritematoso sistémico (LES) presentan una elevada morbi-mortalidad asociada a las manifestaciones cardiopulmonares que se desarrollan durante la evolución de la enfermedad. En este sentido, se han descrito dos picos de incidencia de mortalidad de esta enfermedad inmunológica. El primer pico está relacionado a la actividad de la enfermedad y se observa durante los tres primeros años posteriores al diagnóstico. El segundo pico está relacionado principalmente a las complicaciones cardiopulmonares y se presenta entre los 4 y 20 años posteriores al diagnóstico. Si bien la mortalidad temprana ha disminuido gracias a un mayor conocimiento de la fisiopatología de la enfermedad y al uso de terapias inmunosupresoras, la mortalidad tardía presenta un aumento progresivo de su frecuencia a pesar del avance en el manejo terapéutico. El estudio de estas complicaciones se ha convertido en un tema de gran interés debido a su efecto negativo en el pronóstico de los pacientes que las presentan. Algunos estudios epidemiológicos sugieren que los pacientes con LES tienen un mayor riesgo cardiovascular al ser comparados con la población sana. En este sentido, se ha descrito que estos presentan un mayor riesgo de desarrollar eventos cardiovasculares (infarto agudo del miocardio, accidente cerebro vascular y enfermedad arterial periférica) en comparación con la población general. De forma asociada, se ha objetivado que la presencia de manifestaciones pleuro-pulmonares (pleuritis lúpica, neumonitis, hemorragia pulmonar, embolismo pulmonar e hipertensión pulmonar) aumenta la morbimortalidad de los pacientes con LES. El conocimiento adecuado de las complicaciones cardiopulmonares en los pacientes con diagnóstico de lupus eritematoso sistémico permitirá realizar un manejo individualizado y por lo tanto más eficaz, permitiendo disminuir la morbilidad asociada a las citadas complicaciones. El objetivo de este manuscrito es realizar una revisión de la literatura sobre las principales manifestaciones cardiopulmonares, además de analizar la morbilidad y mortalidad asociada a ellas dentro del contexto clínico de los pacientes con LES.

Palabras clave: manifestaciones cardiopulmonares, lupus eritematoso sistémico, morbilidad y mortalidad cardiopulmonar.

\section{Cardiopulmonary morbidity and mortality in patients with systemic lupus erythematosus}

\author{
ABSTRACT \\ Patients with the diagnosis of systemic lupus erythematosus (SLE) have an elevated \\ morbidity and mortality from cardiopulmonary complications that develop during the \\ evolution of the disease. Considering the incidence of the mortality in this immunologic
}


disease, two peaks have been described. The first one is related to the immune activity itself and it is observed in the first three years the diagnosis. The second peak is related to the cardiopulmonary complications and it is observed after 4 to 20 years after diagnosis. Although, early mortality has diminished due to better knowledge of the physiopathology of the disease and to the use of immunosuppressive drugs, late mortality has a progressive increment despite advances in the therapeutic management. There is a great interest in the study of these complications due to the negative impact on the prognosis of the SLE patients. Some epidemiologic studies suggest that patients with SLE have a higher cardiovascular risk than the healthy population. Indeed, they have greater risk of developing cardiovascular events (acute myocardial infarction, cerebrovascular accidents, and peripheral artery disease) in comparison to the general population. The presence of pulmonary complications (pleuritic effusion, pneumonitis, pulmonary hemorrhage, pulmonary embolism, pulmonary hypertension) increases the mortality in SLE patients. The proper knowledge of the cardiopulmonary complications in SLE patients will provide an individual and more effective management allowing a decrease in morbidity and mortality. The aim of this manuscript is to revise the literature on cardiopulmonary complications and their associated morbidity and mortality in SLE patients.

Keywords: cardiopulmonary manifestations, systemic lupus erythematosus, cardiopulmonary morbidity, mortality.

\section{INTRODUCCIÓN}

El Lupus Eritematoso Sistémico (LES) es una enfermedad inflamatoria autoinmune y multisistémica que se caracteriza por presentar diferentes manifestaciones clínicas constitucionales o especificas dependiendo del compromiso orgánico (1-4). Este compromiso orgánico se debe a un estado inflamatorio secundario a las alteraciones tanto del sistema inmune innato como del adaptativo (5-9).

La gravedad de esta enfermedad es variable y se ve influenciada por determinadas características. En este sentido, varios estudios han demostrado que el pronóstico de los pacientes con esta enfermedad está influenciado por la etnia, el género, la edad y factores socioeconómicos como los ingresos económicos, el nivel de educación y el acceso a los servicios de salud (10-14).

En relación a la etnia, se han descrito diferencias en la incidencia y en el curso clínico de la enfermedad según el diferente origen de los pacientes. Varios estudios han objetivado que los individuos de raza negra tienen una incidencia y prevalencia tres veces mayor que los individuos de raza blanca y que además desarrollan la enfermedad de forma más temprana que éstos $(15,16)$. Se ha descrito que el LES tiende a ser una enfermedad más grave en la población de origen no caucásico. Así los pacientes de origen afroamericano, latinoamericano, y orientales presentan formas más graves de la enfermedad con una morbimortalidad aumentada. Con respecto a los pacientes de origen hispánico, se ha demostrado que éstos desarrollan una enfermedad más grave y con peores resultados terapéuticos que sus pares caucásicos (17-19).

En relación a la mortalidad de la enfermedad, se ha descrito un patrón bimodal en su incidencia, objetivándose dos picos de la misma durante la evolución de la enfermedad. El primero se observa durante los tres primeros años posteriores al diagnóstico de la enfermedad, debido principalmente a infecciones, afectación renal y al uso de corticoides. El segundo pico se relaciona principalmente con el desarrollo de aterosclerosis y enfermedad cardiovascular prematura, lo que ocasiona un aumento de la mortalidad dos veces mayor que el descrito en la población general $(1,20)$.

En algunos estudios epidemiológicos se sugiere que los pacientes con LES tienen un elevado riesgo cardiovascular en relación a la población sana. En ellos se ha descrito una mayor incidencia de infarto agudo del miocardio y de accidentes cerebrovasculares, 
además de una mayor mortalidad asociada a dichos eventos (21).

En relación a la morbimortalidad, la patología pleuro-pulmonar es una complicación que conlleva una mayor morbilidad en los pacientes con LES. Si bien la mayoría de los pacientes no desarrollan una intersticiopatía clínicamente evidente, el LES como patología autoinmune es responsable del $2 \%$ de las muertes provocadas por una afectación pulmonar $(2,22)$.

Las manifestaciones cardiovasculares y pleuro-pulmonares aumentan la morbimortalidad de los pacientes con LES, por lo que un mayor conocimiento de estas complicaciones nos permitirá actuar de forma rápida y eficaz, mejorando el pronóstico de los pacientes en etapas incipientes de esta enfermedad inmunológica. Por lo tanto, el objetivo del presente manuscrito es realizar una revisión de la morbimortalidad cardiovascular de los pacientes con lupus eritematoso sistémico.

\section{Etiopatogenia del Lupus Eritematoso Sistémico}

Para poder comprender mejor las complicaciones cardiopulmonares y su morbimortalidad asociada es de suma importancia entender la etiopatogenia de esta enfermedad autoinmune. La patogenia del LES es compleja, por lo que es considerada la enfermedad más representativa de los procesos de naturaleza autoinmunitaria. Se caracteriza por la producción excesiva de autoanticuerpos, formación de complejos inmunes y daño tisular mediado inmunológicamente (23).

La patología tisular en todos los tejidos refleja una variedad de mecanismos inmunes aberrantes, pero el daño tisular en pacientes con LES también puede reflejar procesos no inmunes. Algunos de éstos, como la aterosclerosis temprana, pueden ser iniciados y acelerados a través de mecanismos inmunológicos $(22,24)$.

En el LES, la respuesta inmune adaptativa es aberrante, observándos eanormalidades tanto de las células $T$ como de las células B. Estas anormalidades incluyen defectos en la tolerancia de las células $B$, de las células $T$ helper, así como irregularidades intrínsecas, funcionales y bioquímicas de las células $T(24,25)$. De forma asociada se observa una apoptosis alterada y un clearance de auto-anticuerpos defectuoso $(26,27)$. La suma de todas estas alteraciones resulta en la producción de autoanticuerpos y de complejos inmunes circulantes $(22,27)$.

Teniendo en cuenta lo mencionado en los párrafos previos, el LES puede ser considerado como la enfermedad modelo para la comprensión de la inmunopatogénesis mediada por complejos inmunes. A diferencia de otras enfermedades autoinmunes que son órgano específicas, el LES se caracteriza por la capacidad de afectar a varios órganos. Ciertamente la formación de auto-anticuerpos, el depósito de inmunoglobulinas y la infiltración de los tejidos por células mononucleares son las principales características de esta enfermedad $(25,27)$.

Las respuestas antígeno-específicas y las antígeno no específicas son importantes en la patogenia de la enfermedad, y se manifiestan a través de un amplio rango de mecanismos patológicos, que incluyen la necrosis fibrinoide, los cuerpos de hematoxilina, la injuria vascular, la disrupción de la unión dermo-epidermica de la piel, y el desarrollo de glomerulonefritis.

\section{Factores Genéticos y No genéticos asociados a la patogenia del LES}

El lupus eritematoso sistémico es una enfermedad compleja de etiología desconocida, en la que están implicados factores genéticos y no genéticos. Entre los factores no genéticos, el género, el uso de determinados fármacos, la exposición a ciertos patógenos así como la luz ultravioleta, se han asociado al desarrollo de esta enfermedad.

En relación al género, se ha sugerido que las hormonas femeninas tienen un rol importante en esta enfermedad, ya que el $90 \%$ de los pacientes con lupus son 
mujeres. De forma asociada, se ha estipulado que las hormonas femeninas podrían tener un efecto importante en el desarrollo del LES mientras que las hormonas masculinas podrían tener un rol protector para el desarrollo de esta enfermedad $(28,29)$.

Existen determinados fármacos que pueden causar una variante del Lupus llamado "Lupus inducido por drogas". En este sentido, existe una relación establecida con la procainamida, la hidralazina y la quinidina. Las principales manifestaciones asociadas al lupus inducido por drogas son las manifestaciones dérmicas y articulares, siendo las características renales y neurológicas poco frecuentes $(22,28,30,31)$.

Los virus han sido uno de los factores más estudiados en el LES, presentando actualmente un rol importante, como probable factor ambiental que desencadena la enfermedad. En este sentido, se han descrito antecedentes de enfermedades virales al inicio del lupus o inmediatamente antes de una recaída en los pacientes con enfermedad establecida. El virus del Epstein Barr (EBV) es considerado un patógeno importante ya que se ha demostrado una asociación temporal entre el inicio del Lupus y la infección por este agente (32).

Por otro lado, la radiación ultravioleta es el factor ambiental con una asociación más robusta con la patogenia del LES. Un ejemplo de la importancia de este factor es que actualmente la fotosensibilidades considerada como uno de los criterios del Colegio Americano de Reumatología para la clasificación de la enfermedad $(33,34)$.

Los factores genéticos poseen un papel importante en el desarrollo del LES. Se ha determinado que el rango de concordancia es del $25 \%$ para gemelos monocigóticos y aproximadamente $2 \%$ para gemelos dicigóticos (35). Estos rangos indican que la contribución genética es importante, pero no es suficiente para causar el LES. Se han identificado varios genes que probablemente contribuyen al Lupus gracias al estudio del genoma de familias con varios miembros afectos de LES.

Los genes del Complejo Mayor de la Histocompatibilidad (CMH), particularmente el $H L A 1, B 8$, y $D R 3$ han sido relacionados con la patogenia del LES. La respuesta del Linfocito $\mathrm{T}$ a un antígeno se inicia cuando un receptor molecular sobre la superficie del linfocito reconoce el complejo formado por el antígeno y un péptido del $\mathrm{CMH}$ en la superficie de la célula presentadora de antígeno (36).

Además de la asociación con el locus del $H L A-D R$, se han identificado otras asociaciones como la del STAT 4 (signal transducer and activator of transcription 4), PTPN 22 (protein tyrosine phosphatase non receptor type 22), ITGAM (integrin alpha M) entre otros (37-40). El STAT 4 fue el primer gen en quien se ha reportado una asociación tanto con el LES como con la Artritis Reumatoide. Esta asociación ha sido confirmada por numerosos estudios incluyendo un estudio de genoma completo (40).

\section{Manifestaciones cardiopulmonares en los pacientes con LES Manifestaciones Pulmonares}

Como se ha explicado previamente, el LES puede afectar a múltiples órganos vitales lo que a su vez actúa en detrimento de la sobrevida a largo plazo de estos pacientes. En la Figura 1 se puede observar como la presencia de manifestaciones cardiovasculares disminuye la supervivencia de los pacientes con LES a largo plazo. 


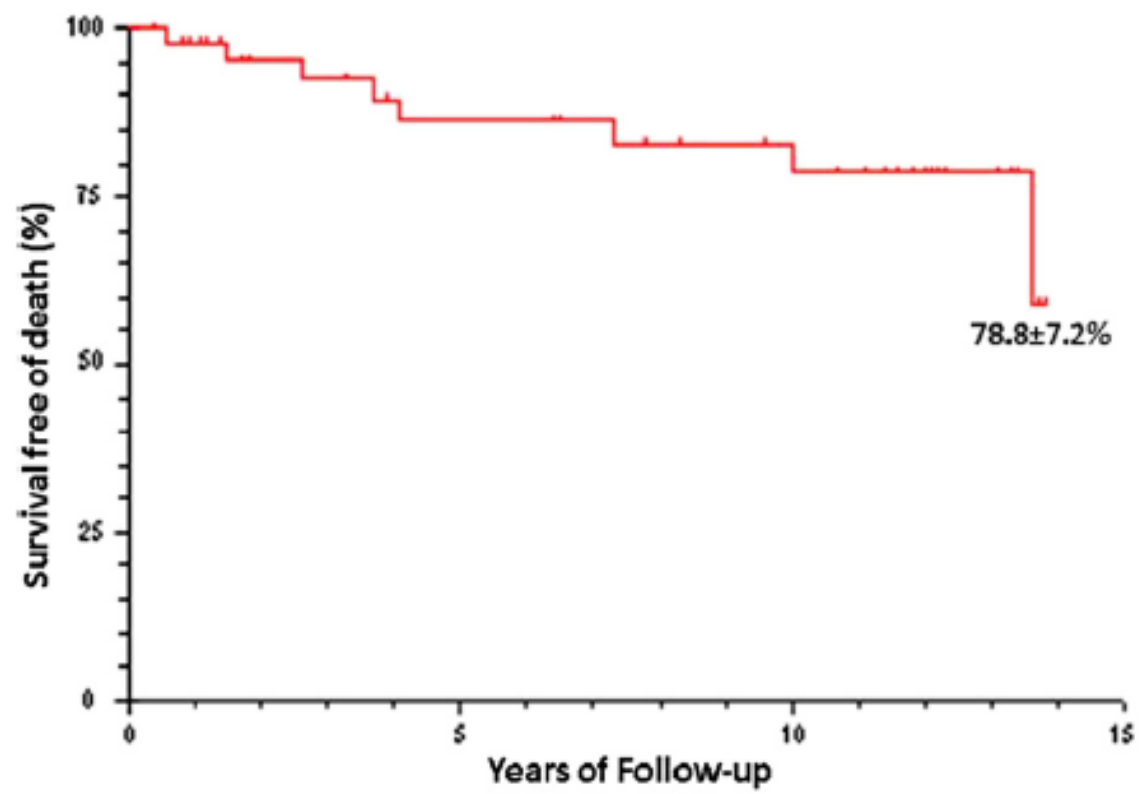

Figura 1. Curva de sobrevida libre de mortalidad de Kaplan-Meier en el seguimiento a largo plazo hasta 13,6 años. Con permiso de Fernandez de Godoy M, et al. (65).

Las manifestaciones pleuro-pulmonares incluyen a la pleuritis lúpica, la neumonitis tanto aguda como crónica, la hemorragia pulmonar, el embolismo pulmonar y la hipertensión pulmonar. Las manifestaciones respiratorias se presentan en cerca de un $25 \%$ de los pacientes con LES y pueden ser clasificadas en primarias (debidas a la enfermedad) y en secundarias a otras patologías $(32,41)$

La afectación pleural, además de ser un criterio de clasificación del LES, es la manifestación respiratoria más frecuentemente observada(32). Ha sido reportada en el $30-60 \%$ de los pacientes con LES $(32,42)$, si bien hasta un $93 \%$ ha sido descrito en estudios basados en autopsias. El derrame pleural en el LES se caracteriza por ser bilateral y de pequeño volumen. Se presenta clínicamente con dolor pleurítico, tos y disnea, si bien en algunos casos pueden ser asintomáticos $(32,43)$. El análisis del líquido pleural, presenta características de exudado, que puede ser inicialmente de predominio neutrofílico para a los pocos días volverse predominantemente linfocítico $(42,43)$.

La neumonitis presenta una menor frecuencia, y se ha descrito en un $0-14 \%$ de los pacientes (32). Esta puede tener una presentación aguda o crónica. La primera se manifiesta generalmente como una recaída de la enfermedad multisistémica; los pacientes se presentan clínicamente con tos, disnea, dolor torácico, fiebre e inclusive hemoptisis. La importancia de esta complicación radica en la alta morbimortalidad que conlleva. Se ha descrito que hasta un $50 \%$ de los pacientes que la padecen fallece, y el grupo de pacientes que sobrevive presenta cronificación de la complicación pulmonar (32).

En relación a la neumonitis lúpica de evolución crónica generalmente se presenta como una enfermedad pulmonar intersticial que se caracteriza por tos no productiva, disnea de esfuerzo, fatiga y rales basales (42). Puede producirse como una complicación de la neumonitis aguda o puede presentarse de forma independiente a ésta (43). Los estudios de imagen revelan un infiltrado intersticial, con imágenes en vidrio deslustrado y la presencia de opacidades reticulares basales (43). Es importante considerar que si bien la mayoría de los pacientes no desarrolla una intersticiopatía clínicamente evidente, el LES como patología autoinmune es responsable del $2 \%$ de las muertes provocadas por una 
afectación pulmonar (44).

La hemorragia alveolar es una complicación poco frecuente que implica una gravedad importante ya que se asocia con una elevada mortalidad que varía entre el $50-90 \%$. Se presenta característicamente con disnea de inicio brusco, tos, fiebre, infiltrados pulmonares con predominio en los lóbulos inferiores y una caída brusca de la hemoglobina. La hemoptisis es una manifestación típica de la enfermedad, pero se presenta en el $40-60 \%$ de los casos, por lo que un porcentaje no despreciable de los pacientes no la presentará. Clásicamente se ha descrito la presencia de anemia, condensaciones pulmonares y hemoptisis en esta complicación (43).

La hipertensión pulmonar puede ocurrir en el LES debido a la propia enfermedad o como consecuencia de complicaciones como el embolismo pulmonar, la enfermedad valvular cardiaca o la enfermedad pulmonar intersticial. Se ha descrito su presencia en un porcentaje que varía entre el $5-14 \%$ de los pacientes con LES (45). El desarrollo de esta complicación se ha asociado frecuentemente a la presencia de anticuerpos antifosfolípidos, pero es importante recalcar que las complicaciones tromboembólicas en el LES pueden estar presentes con o sin estos anticuerpos (45). A modo de resumen en la tabla 1 se presentan las principales complicaciones pleuropulmonares descritas en el LES.

Tabla 1. Manifestaciones pleuro-pulmonares más frecuentes en el LES

\begin{tabular}{ll}
\hline Enfermedad Pleural & Derrame pleural \\
\hline \multirow{3}{*}{ Enfermedad Parenquimatosa } & Neumonitis lúpica aguda \\
& Hemorragia alveolar difusa \\
& Neumonitis lúpica crónica \\
Afectación vascular & Hipoxemia aguda \\
& Embolismo pulmonar \\
Afectación de las vías aéreas & Hipertensión arterial pulmonar \\
& Enfermedad obstructiva \\
\hline
\end{tabular}

Tabla modificada de Pego-Reinosa et al. (41)

\section{Manifestaciones Cardiovasculares}

La afectación cardiovascular es una de las causas más importantes de morbilidad y mortalidad en los pacientes con diagnóstico de LES. En este sentido se ha descrito que los pacientes con LES presentan un mayor riesgo para padecer un infarto agudo de miocardio y/o un accidente cerebrovascular comparado con el riesgo que presenta la población general (46) (Figura 2). Los pacientes jóvenes con LES tienen un riesgo aumentado a tener un evento cardiovascular con respecto a las personas sanas de la misma edad; y este riesgo aumenta a medida que aumenta la edad. 


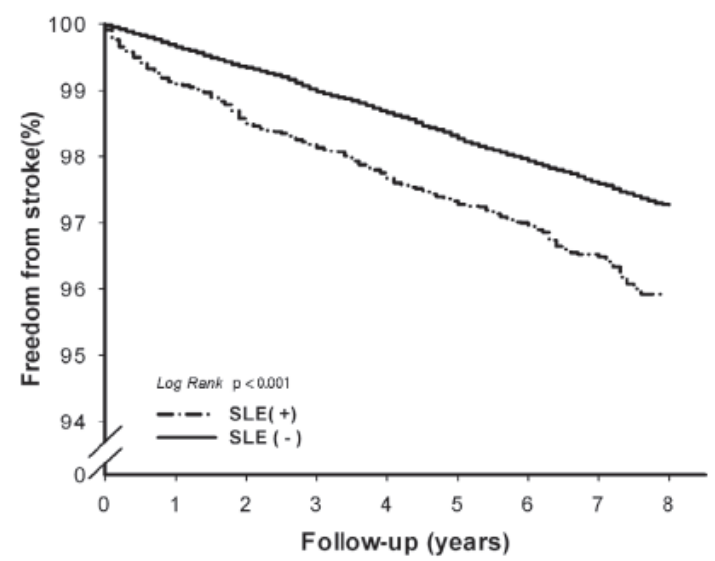

Figura 2. Curva de Kaplan-Meier libre de accidente cerebrovascular isquémico en pacientes con y sin lupus eritematoso sistémico a ocho años de seguimiento. Existe una diferencia estadísticamente significativa $(p<0.001)$. Con permiso de Chiu CC et al. (66).

Además de los factores de riesgo cardiovasculares tradicionales (por ej. la dislipidemia, el tabaquismo, la edad avanzada, la hipertensión arterial el sexo masculino, etc.), se han descrito nuevos factores de riesgo (genéticos, asociados a la inmunidad y asociados con el grado de inflamación) asociados a la propia enfermedad. Tanto los factores de riesgo cardiovascular tradicionales como los nuevos factores de riesgo, están asociados a un mayor riesgo para desarrollar arteriosclerosis y presentar un evento cardiovascular entre los pacientes con LES (Figura 3 ).

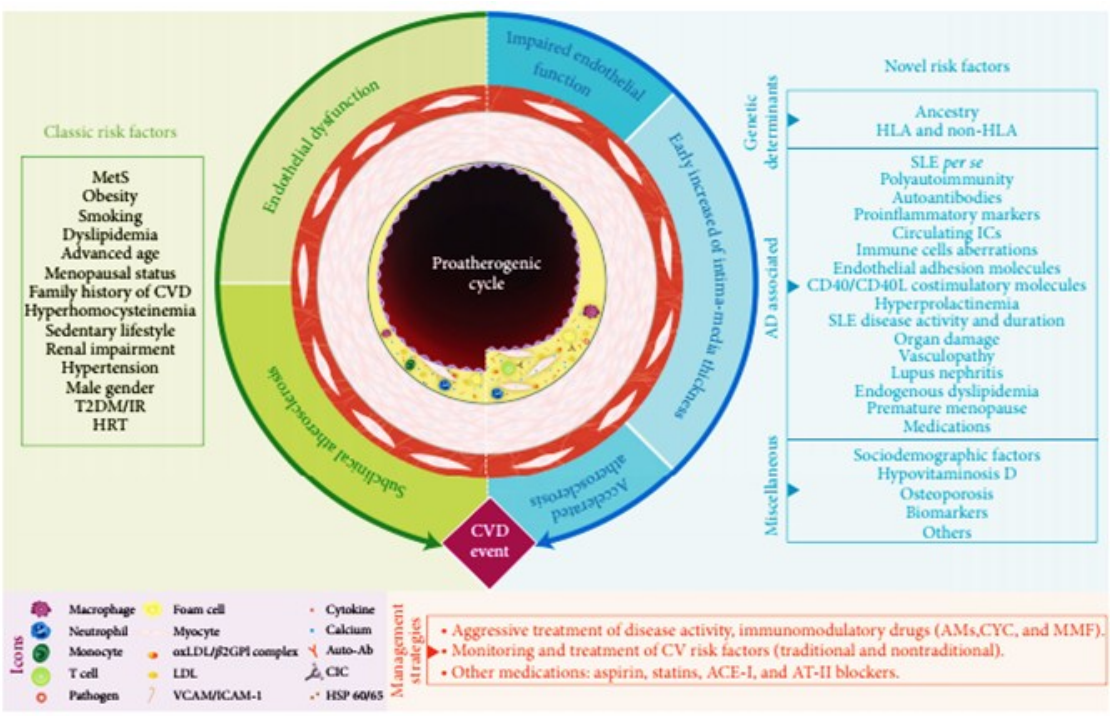

Figura 3. Interacción compleja en el mecanismo aterogénico entre los factores de riesgo cardiovascular clásicos y los factores nuevos de riesgo cardiovascular relacionados a la enfermedad inmunológica en el lupus eritematoso sistémico. CVD: cardiovascular disease, SLE: systemic lupus erythematosus, MetS: metabolic syndrome, T2DM: type 2 diabetes mellitus, IR: insulin resistance, HRT: hormone replacement therapy, CIC: Circulating Immune complex, oxLDL/ B2GPI complex: oxidized-low density lipoprotein/B 2 glycoprotein I, HDL: high density lipoprotein, Auto-Ab: auto-antibodies, AMs: antimalarials, CYC: cyclophosphamide, AZA: azathioprine, MMF: mycophenolatemofetil, ACEI: angiotensin-converting enzyme inhibitors, AT-II blockers: angiotensin II receptor blockers. Con permiso de Amaya-Amaya J et al. (67). 
En un estudio realizado en pacientes con LES, el 53\% de éstos tenía más de un factor de riesgo tradicional y de éstos los más frecuentes eran el sedentarismo (70 \%), la obesidad (56\%), la dislipidemia (56\%) y el antecedente de tabaquismo (56\%) (47).

El corazón puede estar comprometido clínicamente en el $50 \%$ de los pacientes con LES (48-50). Todas las estructuras cardiacas pueden estar afectadas, desde el pericardio, el miocardio, el endocardio, las arterias coronarias hasta el tejido de conducción. Este compromiso puede ser debido al LES o a otra enfermedad sistémica como la hipertensión arterial.

Aunque algunos estudios de necropsia han demostrado un 40-70\% de miocarditis; la miocarditis sintomática es reportada en solo un $5-10 \%$ de los pacientes, sugiriendo que el compromiso subclínico cardiaco es un hallazgo común en el LES (51-53).

En relación a los eventos coronarios, Manzi et al. (50) han reportado que los eventos coronarios fueron 50 veces más frecuentes en los pacientes con LES en el rango etario de 35-44 años en relación a la población sana de la cohorte de Framingham (50). En la cohorte de LES del hospital John Hopkins el riesgo de desarrollar un evento cardiovascular fue de 2,6 veces mayor que para la población de Framingham incluso después de controlar los factores de riesgo para enfermedad cardiovascular tradicionales en este grupo (51).

El síndrome antifosfolipídico está caracterizado por un riesgo incrementado para accidentes cerebrovasculares e infartos de miocardio y por lo tanto, varios estudios han intentado determinar si la presencia de anticuerpos antifosfolipídicos puede ser un factor de riesgo independiente para enfermedad cardiovascular. Se ha observado que la presencia de anticuerpos antifosfolipídicos estuvo significativamente asociada con un incremento del riesgo de 4 veces de tener un evento cardiovascular en un promedio de tiempo de aproximadamente 6 años (54-57).

Adicionalmente, se ha observado que existe una asociación entre el síndrome nefrótico y una prevalencia aumentada de arteriosclerosis no sintomática en pacientes lúpicos diagnosticados antes de los 16 años; y que los niveles aumentados de creatinina se asociaron a un mayor riesgo de padecer coronariopatía $(58,59)$.

\section{Mortalidad en el LES}

El rango de sobrevida de los pacientes con LES se ha incrementado notablemente en las ultimas 5 décadas, pasando de menos del $50 \%$ a los 5 años en 1955 al $85 \%$ a los 10 años (2). Esta mejoría en la supervivencia de los pacientes con LES es el resultado del aumento de la sobrevida global de la población en general, los avances en las modalidades terapéuticas, el uso más juicioso de las terapias existentes, y a un cambio en los factores de riesgo de mortalidad. A pesar del alentador progreso, se ha descrito que los pacientes diagnosticados con LES y seguidos en varios centros de EEUU tienen el riesgo de mortalidad 2,4 veces mayor que el de la población general. Este incremento en la mortalidad es el resultado de las infecciones, las enfermedades cardiopulmonares y el daño irreversible de los órganos blanco (2).

Los eventos cardiovasculares son una complicación bien conocida del LES. En 1972, Urowitz et al. (1) describieron un patrón de mortalidad con dos picos de incidencia en los pacientes con LES. El primer pico se presentaría durante los tres primeros años posteriores al diagnóstico de la enfermedad. Las principales causas de muerte serían secundarias a la actividad del LES, a las infecciones, la afectación renal y las altas dosis de corticoides administrados durante las primeras fases de la enfermedad. El segundo pico se presentaría de forma más tardía, entre los 4 y los 20 años posteriores al diagnóstico. La principal causa de mortalidad tardía estaría relacionada principalmente con los eventos cardiovasculares. Desde entonces se ha despertado un interés creciente tanto en la epidemiología como la fisiopatología de la enfermedad cardiovascular entre los pacientes con LES. 
Aunque la mortalidad de los pacientes con LES ha mejorado en los últimos 30 años gracias a los avances en el tratamiento y a una mejor comprensión de los mecanismos patológicos, se ha objetivado que la mortalidad debido a la enfermedad cardiovascular aumenta $(3,4,60)$. Los mecanismos subyacentes asociados a una aterosclerosis acelerada en el LES son complejos y son áreas de investigación exhaustiva en los últimos años. Aunque los riesgos tradicionales como la dislipidemia y el tabaquismo han sido demostrados como factores predictores de riesgo cardiovascular en pacientes con LES, el LES por sí mismo es un factor de riesgo independiente para enfermedad cardiovascular (Figura 4) (3,61-64).

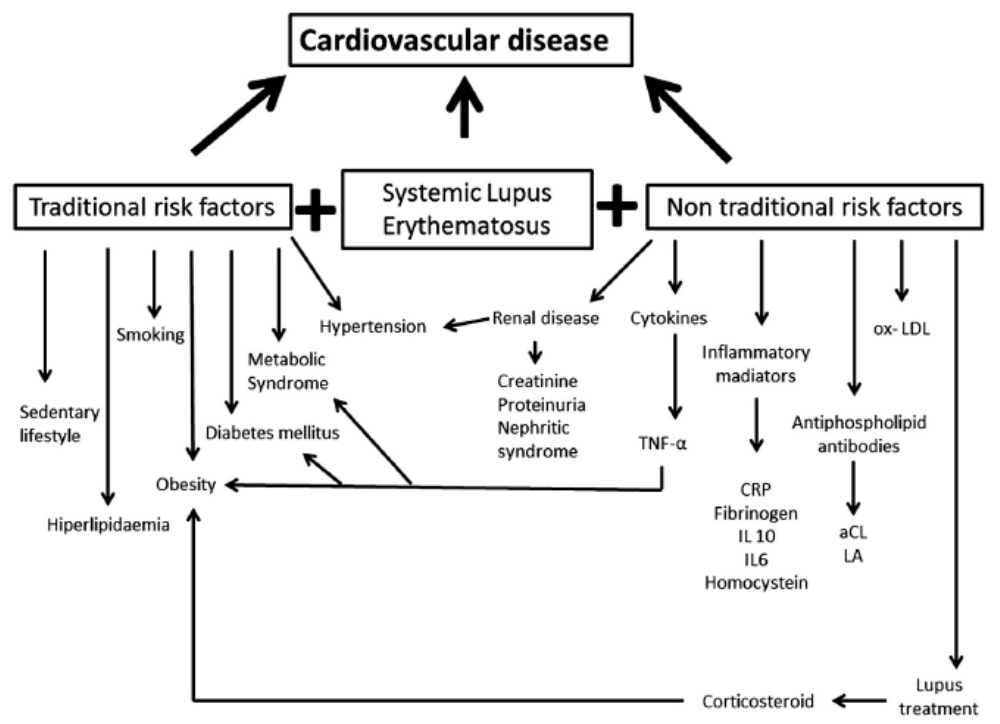

Figura 4. Interacción de los factores de riesgo tradicionales, y no tradicionales en los pacientes con lupus eritematoso sistémico en la producción de enfermedades cardiovasculares. Con permiso de Sinicato NA, et al. (68).

\section{CONCLUSIÓN}

La evidencia científica indica que existe un claro y significativo aumento de eventos cardiopulmonares en los pacientes con LES. Los eventos cardiovasculares más frecuentes son la hipertensión arterial, seguida de los accidentes cerebrovasculares y el infarto agudo de miocardio. El conocimiento adecuado de las complicaciones cardiopulmonares que aumentan considerablemente la mortalidad en el LES nos permitirá actuar más rápidamente y mejorar nuestro manejo terapéutico individualizado en etapas incipientes de esta enfermedad inmunológica.

\section{REFERENCIAS BIBLIOGRÁFICAS}

1.Urowitz MB, Bookman AA, Koehler BE, Gordon DA, Smythe HA, Ogryzlo MA. The bimodal mortality pattern of systemic lupus erythematosus. Am J Med. 1976; 60(2):2215.

2.Bernatsky S, Boivin JF, Joseph L, Manzi S, Ginzler E, Gladman DD, et al. Mortality in systemic lupus erythematosus. Arthritis Rheum. 2006; 54(8):2550-7.
3.Bjornadal L, Yin L, Granath F, Klareskog L, Ekbom A. Cardiovascular disease a hazard despite improved prognosis in patients with systemic lupus erythematosus: Results from a swedish population based study 1964-95. J Rheumatol. 2004;31(4):713-9.

4.Arnaud L, Mathian A, Adoue D, Bader-Meunier $B$, Baudouin V, Belizna $C$, et al. Dépistage et prise en charge du risque cardiovasculaire au cours du lupus systémique: Elaboration de 
recommandations pour la pratique clinique, à partir d'une analyse de la littérature et de I'avis d'experts. La Revue de Médecine Interne. 2015; 36(6):372-80.

5.Arbuckle MR, McClain MT, Rubertone MV, Scofield RH, Dennis GJ, James JA, et al. Development of autoantibodies before the clinical onset of systemic lupus erythematosus. N Engl J Med. 2003;349(16):1526-33.

6.Alarcon-Segovia D, Alarcon-Riquelme $M$. Etiopathogenesis of systemic lupus erythematosus: A tale of three troikas. In: Latiha $\mathrm{R}$, editor. Systemic lupus erythematosus. New York: Academic Press; 1999. p. 55-65.

7.Drenkard C, Alarcon-Segovia D. The new prognosis of systemic lupus erythematosus: treatment-free remission and decreased mortality and morbidity. Isr Med Assoc $\mathrm{J}$. $2000 ; 2(5): 382-7$.

8.Drenkard C, Villa AR, Garcia-Padilla C, Perez -Vazquez ME, Alarcon-Segovia D. Remission of systematic lupus erythematosus. Medicine (Baltimore). 1996; 75(2):88-98.

9.Gladman DD. Prognosis and treatment of systemic lupus erythematosus. Curr Opin Rheumatol. 1996; 8(5):430-7.

10.Pons-Estel BA, Catoggio LJ, Cardiel MH, Soriano ER, Gentiletti S, Villa AR, et al. The GLADEL multinational Latin American prospective inception cohort of 1,214 patients with systemic lupus erythematosus: Ethnic and disease heterogeneity among Hispanics. Medicine (Baltimore). 2004; 83(1):1-17.

11.Devins GM, Edworthy SM. Illness intrusiveness explains race-related quality-of-life differences among women with systemic lupus erythematosus. Lupus. 2000; 9(7):534-41.

12.Ginzler EM, Diamond HS, Weiner M, Schlesinger M, Fries JF, Wasner C, et al. A multicenter study of outcome in systemic lupus erythematosus: I. Entry variables as predictors of prognosis. Arthritis Rheum. 1982; 25(6):601-11.

13. Halberg P, Alsbjorn B, Balslev JT, Lorenzen I, Gerstoft J, Ullman S, et al. Systemic lupus erythematosus: Follow-up study of 148 patients. II: Predictive factors of importance for course and outcome. Clin Rheumatol. 1987; $6(1): 22-6$.

14.Karlson EW, Daltroy LH, Lew RA, Wright EA, Partridge AJ, Fossel $A H$, et al. The relationship of socioeconomic status, race, and modifiable risk factors to outcomes in patients with systemic lupus erythematosus. Arthritis Rheum. 1997; 40(1):47-56.

15. McCarty DJ, Manzi S, Medsger TA Jr, Ramsey-Goldman R, LaPorte RE, Kwoh CK. Incidence of systemic lupus erythematosus: Race and gender differences. Arthritis
Rheum. 1995;38(9):1260-70.

16. Molokhia M, Hoggart C, Patrick AL, Shriver M, Parra $E$, Ye J, et al. Relation of risk of systemic lupus erythematosus to west African admixture in a Caribbean population. Hum Genet. 2003;112(3):310-8.

17. Alarcon GS, Roseman J, Bartolucci AA, Friedman AW, Moulds JM, Goel N, et al. Systemic lupus erythematosus in three ethnic groups: II. Features predictive of disease activity early in its course. LUMINA Study Group. Lupus in minority populations, nature versus nurture. Arthritis Rheum. 1998; 41(7):1173-80.

18. Reveille JD, Moulds JM, Ahn C, Friedman AW, Baethge B, Roseman J, et al. Systemic lupus erythematosus in three ethnic groups: I. The effects of HLA class II, C4, and CR1 alleles, socioeconomic factors, and ethnicity at disease onset. LUMINA Study Group. Lupus in minority populations, nature versus nurture. Arthritis Rheum. 1998; 41(7):1161-72.

19.Peralta-Ramirez MI, Jimenez-Alonso J, GodoyGarcia JF, Perez-Garcia M. The effects of daily stress and stressful life events on the clinical symptomatology of patients with lupus erythematosus. Psychosom Med. 2004; 66(5):788-94.

20.Fraser PA, Ding WZ, Mohseni M, Treadwell EL, Dooley MA, St Clair EW, et al. Glutathione Stransferase $M$ null homozygosity and risk of systemic lupus erythematosus associated with sun exposure: A possible gene-environment interaction for autoimmunity. J Rheumatol. 2003; 30 (2):276-82.

21.Schoenfeld SR, Kasturi S, Costenbader KH. The epidemiology of atherosclerotic cardiovascular disease among patients with SLE: A systematic review. Semin Arthritis Rheum. 2013;43 (1):77-95.

22.Leathart L, Respiratory disease: Task force report on problems, research approaches, needs. $\mathrm{Br} J$ Ind Med. 1974; 31(2):172-3

23. Rekvig OP, Van der Vlag J. The pathogenesis and diagnosis of systemic lupus erythematosus: Still not resolved. Semin Immunopathol. 2014; 36(3):301-11.

24. Mohan C, Putterman C. Genetics and pathogenesis of systemic lupus erythematosus and lupus nephritis. Nat Rev Nephrol. 2015;11 (6):329-41.

25.Lisnevskaia L, Murphy G, Isenberg D. Systemic lupus erythematosus. Lancet. 2014;384 (9957): 1878-88

26.Munoz LE, Gaipl US, Franz S, Sheriff A, Voll $\mathrm{RE}$, Kalden JR, et al. SLE--a disease of clearance deficiency?. Rheumatology (Oxford). 2005; 44 (9):1101-7.

27. Wahren-Herlenius $M$, Dorner T. Immunopathogenic mechanisms of systemic autoimmune disease. Lancet. 2013;382(9894):819-31. 
28. Klemperer P, Pollack AD, Baehr G. Diffuse collagen disease: Acute disseminated lupus erythematosus and diffuse scleroderma. JAMA. $1942 ; 119(4): 331-2$.

29. Cutolo M, Sulli A, Straub RH. Estrogen metabolism and autoimmunity. Autoimmun Rev. 2012; 11(6-7):A460-4.

30. Mason LJ, Isenberg D. The pathogenesis of systemic lupus erythematosus. In: Davidson AM, Cameron JS, Grunfeld JP, editors. Oxford textbook of clinical nephrology. Oxford: Oxford University Press; 2005. p.809-29.

31.Rubin R. Drug induced lupus. In: Wallace DJ, Hahn $\mathrm{BH}$, editors. Dubois' lupus erythematosus. 6th ed. Philadelphia: Lippincott Williams \& Wilkins; 2002. p.885-916.

32.Villa Blanco I, Calvo Alén J. Lupus eritematoso sistémico. In: Alperi Lopez $M$, Balsa Criado A, Blanco A, Hernandez Cruz B, Medina Luezas J, Muñoz-Fernández S, et al., editors. Manual SER de enfermedades reumáticas. 6a ed. Madrid: Elsevier; 2014. p.335-62.

33.Tan EM, Cohen AS, Fries JF, Masi AT, McShane DJ, Rothfield NF, et al. The 1982 revised criteria for the classification of systemic lupus erythematosus. Arthritis Rheum. 1982;25(11):1271-7.

34. Hochberg MC. Updating the American College of Rheumatology revised criteria for the classification of systemic lupus erythematosus. Arthritis Rheum. 1997;40(9):1725.

35.Sullivan KE. Genetics of systemic lupus erythematosus: Clinical implications. Rheum Dis Clin North Am. 2000; 26(2):229-56.

36. Walport MJ. Complement and systemic lupus erythematosus. Arthritis Res. 2002;4 (Suppl 3):S279-93.

37.Lee YH, Bae SC. Association between the functional ITGAM rs1143679 G/A polymorphism and systemic lupus erythematosus/ lupus nephritis or rheumatoid arthritis: an update meta-analysis. Rheumatol Int. 2015; 35 (5):815-23.

38. Ostanek L, Ostanek-Panka M, BobrowskaSnarska D, Binczak-Kuleta A, Fischer K, Kaczmarczyk M, et al. PTPN22 1858C>T gene polymorphism in patients with SLE: association with serological and clinical results. Mol Biol Rep. 2014;41(9):6195-200.

39. Harley JB, Alarcon-Riquelme ME, Criswell LA, Jacob CO, Kimberly RP, Moser KL, et al. Genome-wide association scan in women with systemic lupus erythematosus identifies susceptibility variants in ITGAM, PXK, KIAA1542 and other loci. Nat Genet. 2008; 40(2):20410.

40.Remmers EF, Plenge RM, Lee AT, Graham RR, Hom G, Behrens TW, et al. STAT4 and the risk of rheumatoid arthritis and systemic lupus erythematosus. N Engl J Med. 2007;357 (10): 977-86.

41.Pego-Reigosa JM, Medeiros DA, Isenberg DA. Respiratory manifestations of systemic lupus erythematosus: Old and new concepts. Best Pract Res Clin Rheumatol. 2009;23(4):469-80.

42. Memet B, Ginzler EM. Pulmonary manifestations of systemic lupus erythematosus. Semin Respir Crit Care Med. 2007;28 (4):441-50.

43.Pena Gonzalez A, Rodriguez Casal P, Gonzalez Barcala FJ. Manifestaciones pulmonares del lupus eritematoso sistémico. Pneuma. 2010; 6(3):109-14.

44.Kao AH, Manzi S. How to manage patients with cardiopulmonary disease?. Best Pract Res Clin Rheumatol. 2002;16(2):21127.

45.Pan TL, Thumboo J, Boey ML. Primary and secondary pulmonary hypertension in systemic lupus erythematosus. Lupus. $2000 ; 9(5): 338-42$.

46. Doria A, Iaccarino $L$, Sarzi-Puttini P, Atzeni $F$, Turriel $M$, Petri $M$. Cardiac involvement in systemic lupus erythematosus. Lupus. $2005 ; 14(9): 683-6$.

47.Wijetunga $M$, Rockson $S$. Myocarditis in systemic lupus erythematosus. Am J Med. 2002; 113(5):419-23.

48. Makaryus JN, Catanzaro JN, Goldberg S, Makaryus AN. Rapid progression of atrioventricular nodal blockade in a patient with systemic lupus erythematosus. Am J Emerg Med. 2008;26(8):967.e5-7.

49. Moder KG, Miller TD, Tazelaar HD. Cardiac involvement in systemic lupus erythematosus. Mayo Clin Proc. 1999; 74(3):275-84.

50.Manzi S, Meilahn EN, Rairie JE, Conte CG, Medsger TA, Jr, Jansen-McWilliams L, et al. Age-specific incidence rates of myocardial infarction and angina in women with systemic lupus erythematosus: Comparison with the Framingham study. Am J Epidemiol. $1997 ; 145(5): 408-15$.

51. Magder LS, Petri M. Incidence of and risk factors for adverse cardiovascular events among patients with systemic lupus erythematosus. Am J Epidemiol. 2012;176(8):708 $-19$.

52. Navarra SV, Guzman RM, Gallacher AE, Hall S, Levy RA, Jimenez RE, et al. Efficacy and safety of belimumab in patients with active systemic lupus erythematosus: a randomised, placebo-controlled, phase 3 trial. Lancet. 2011;377(9767):721-31.

53.Abu-Shakra M, Novack V. Mortality and multiple causes of death in systemic lupus 
erythematosus -- role of the death certificate. J Rheumatol. 2012;39(3):458-60.

54.Zandman-Goddard G, Shoenfeld Y. SLE and infections. Clin Rev Allergy Immunol. 2003;25 (1):29-40.

55. Mok CC, Kwok CL, Ho LY, Chan PT, Yip SF. Life expectancy, standardized mortality ratios, and causes of death in six rheumatic diseases in Hong Kong, China. Arthritis Rheum. 2011;63(5):1182-9.

56.Smolen JS, Strand V, Cardiel M, Edworthy S, Furst D, Gladman D, et al. Randomized clinical trials and longitudinal observational studies in systemic lupus erythematosus: Consensus on a preliminary core set of outcome domains. J Rheumatol. 1999;26(2):504-7.

57.Strand V, Gladman D, Isenberg D, Petri M, Smolen J, Tugwell P. Outcome measures to be used in clinical trials in systemic lupus erythematosus. J Rheumatol. 1999;26(2):490-7.

58.Strand V. Clinical trial design in systemic lupus erythematosus: Lessons learned and future directions. Lupus. 2004;13(5):406-11.

59.Boers M, Brooks P, Simon LS, Strand V, Tugwell P. OMERACT: An international initiative to improve outcome measurement in rheumatology. Clin Exp Rheumatol. 2005;23(5 Suppl 39):S10-3.

60. Neville C, Clarke AE, Joseph L, Belisle P, Ferland D, Fortin PR. Learning from discordance in patient and physician global assessments of systemic lupus erythematosus disease activity. J Rheumatol. 2000;27(3):675-9.

61.Chang ER, Abrahamowicz M, Ferland D, Fortin PR. Organ manifestations influence differently the responsiveness of 2 lupus disease activity measures, according to patients' or physicians' evaluations of recent lupus activity. J Rheumatol. 2002;29(11):2350-8.

62. Wang C, Mayo NE, Fortin PR. The relationship between health related quality of life and disease activity and damage in systemic lupus erythematosus. J Rheumatol. 2001;28(3):525 -32 .
63.Stoll T, Sutcliffe N, Mach J, Klaghofer R, Isenberg DA. Analysis of the relationship between disease activity and damage in patients with systemic lupus erythematosus--a 5-yr prospective study. Rheumatology (Oxford). 2004;43(8):1039-44.

64.Esdaile JM, Abrahamowicz M, Grodzicky T, Li Y, Panaritis C, du Berger $\mathrm{R}$, et al. Traditional framingham risk factors fail to fully account for accelerated atherosclerosis in systemic lupus erythematosus. Arthritis Rheum. 2001;44(10):2331-7.

65.Fernández de Godoy $M$, Matsuura de Oliveira C, Alves V, Abreu LC, Valenti VE, Pires AC, et al. Long-term cardiac changes in patients with systemic lupus erithematosus. BMC Research Notes 2013;6:171-7.

66.Chiu CC, Huang CC, Chan WL, Chung CM, Huang $\mathrm{PH}$, Lin $\mathrm{S}$ j et al. Increased risk of ischemic stroke in patients with systemic lupus erithematosus: A nationwide populationbased study. Intern Med 2012;51:17-21.

67. Amaya-Amaya J, Sarmiento-Monroy JC, Caro-Moreno J, Molano-González N, Mantilla RD, Rojas-Villarraga A, et al. Cardiovascular disease in Latin American patients with systemic lupus erithematosus: A cross-sectional study and a systematic review. Autoinmune

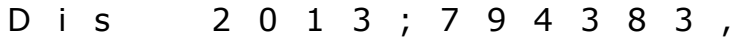
doi: $10.1155 / 2013 / 794383$.

68.Sinicato NA, Da Silva Cardozo PA, Appenzeller S. Risk factors in cardiovascular disease in systemic lupus erithematosus. Curr Cardiol Rev 2013;9:15-19. 